been conducted in an untested context. Also, the investigated public universities can enhance their knowledge management Practices by implementing knowledgeoriented leadership style.

Keywords: Knowledge Management, Public Universities, Knowledge Creation, Bureaucratic Culture, knowledge-oriented Leadership

\title{
Organizational Factors Affecting Knowledge Sharing Capabilities: A Study with Portuguese Health Professionals
}

\author{
Maria Helena Almeida ${ }^{1}$, Alejandro Ramos ${ }^{2}$, Maria José Sousa ${ }^{3}$, \\ Carina Martinho Santos ${ }^{4}$ and Ana Paula Fontes ${ }^{4}$ \\ ${ }^{1}$ Universidade do Algarve, participant member of Research Centre for \\ Tourism, Sustainability and Well-being - CinTurs, Faro, Portugal \\ ${ }^{2}$ Universidad de Málaga, Spain \\ ${ }^{3}$ ISCTE- Instituto Universitário de Lisboa, Portugal \\ ${ }^{4}$ HPA Health Group, Faro, Portugal
}

halmeida@ualg.pt; maria.jose.sousa@iscte-iul.pt; aorgambidez@uma.es anafontes@grupohpa.com; carinafmartinho86@gmail.com

DOI: 10.34190/EKM.20.153

\begin{abstract}
The importance of knowledge management in the healthcare sector has been highlighted in an attempt to expand the speed of new digital technologies to establish external collaboration, employee engagement, networking, and customer service, providing at the same time new challenges for the overall education system. Kim and Lee's empirical study, supported on Davenport and Prusak's framework, evaluated to what extent i) organizational culture (ii) organizational structure, (iii) application of information technology, influence sharing knowledge capabilities in public organizations in South Korea. The goal is to validate Kim and Lee's instrument, trying to replicate the theoretical model suggested by them but not fully validated, with 203 health professionals from the Algarve region (southern Portugal). The data were submitted to an exploratory and confirmatory factor analysis with (STATA), in order to meet a criterion-related validity. Results show the effect of "cultural organizational" through the facets "visions and objectives," "trust," and "social networks" on KSC. The variables "organizational structure" and "information technology" provided no statistical support because they were not significantly associated with employee's knowledge-sharing capabilities. Small
\end{abstract}


sample size and perceptual measures are only some of several limitations. Future studies must prepare a systematic literature review and use the results of this first study in order to find out a valid model.

Keywords: Knowledge, Management, healthcare, organizational culture, organizational structure, information technology application

\title{
A Framework for Enhancing the Sharing of Teaching Practices among University Instructors
}

\author{
Nouf Almujally ${ }^{1,2}$ and Mike Joy ${ }^{1}$ \\ ${ }^{1}$ University of Warwick, Coventry, UK \\ ${ }^{2}$ Princess Nourah Bint Abdulrahman University, Riyadh, Saudi Arabia
}

n.almujally@warwick.ac.uk; M.s.joy@warwick.ac.uk; Naalmujally@pnu.edu.sa DOI: 10.34190/EKM.20.219

\begin{abstract}
This research paper describes a teaching practices management framework (TPMF) that strongly supports sharing processes among instructors, particularly those that can be performed with the help of an information system. The purpose is to allow teachers' accumulated experience to be identified, shared and reused, which can offer significant benefits, including the introduction of teaching innovations. Such innovation can lead to improvements in overall teaching quality and the enhancement of academics' professional development efforts. The development of the TPMF in the present study has been informed by semistructured interviews conducted with 22 instructors working in two universities in Saudi Arabia; these interviews were undertaken to better understand the academics' actual knowledge sharing behaviours in relation to TPs and also to gain a perspective of current knowledge sharing approaches. An inductive coding approach was employed to help the researcher extract themes that were mentioned by the interviewees. It is believed that this framework could be of great assistance to the higher education sector. We further recommend that it could be used as a guideline for developing systems aimed at improving TP sharing among academics.
\end{abstract}

Keywords: knowledge management system, knowledge sharing, higher education institutions, knowledge sharing system, teaching practices 